\title{
Prácticas de Recursos Humanos en la Empresa Familiar: profesionalización y efecto sobre el desempeño
}

\section{Human Resource Practices in the Family Firms: professionalization and effects on firm performance.}

\author{
Antonio J. Carrasco Hernández ${ }^{\mathrm{a}^{*}} \cdot$ Gregorio Sánchez Marín $^{\mathrm{a}} \cdot$ Ángel L. Meroño Cerdán ${ }^{\mathrm{a}}$ \\ ${ }^{a}$ Departamento de Organización de Empresas y Finanzas, Universidad de Murcia
}

\section{A T O S A R T Í C U L O}

Historial:

Recibido 02-07-2014

Aceptado 30-10-2014

Palabras clave:

Empresa familiar

Prácticas de recursos

humanos

Desempeño organizativo

Códigos JEL:

M14, G34

\section{RES UMEN}

La influencia de la familia en las estructuras de gestión del capital humano de la empresa crea un marco único y diferente, con importantes implicaciones en términos de eficiencia. En este trabajo se examina la orientación de las principales prácticas de recursos humanos -selección, formación y retribución- y sus efectos sobre el desempeño organizativo en función de la presencia de la familia en la propiedad y dirección del negocio. Utilizando una muestra de 500 empresas españolas, los resultados indican que en las empresas de dirección familiar se aplican unas prácticas de recursos humanos menos formalizadas y profesionalizadas que en las empresas familiares de dirección externa. Asimismo, se comprueba una contribución de las prácticas de recursos humanos a través de la selección que se encuentra moderada negativamente por el carácter familiar.

\section{A R T I C LE IN F O}

Article history:

Received 02-07-2014

Accepted 30-10-2014

Keywords:

Family Firm

Human resource practices

Firm performance

JEL codes:

M14, G34

\begin{abstract}
A B S T R A C T
Family involvement creates a unique and idiosyncratic setting in managing human capital influencing firm performance. This paper examines the orientation of the main HR practices - selection, training and compensation - and their effects on firm performance based on the presence of the family in ownership and management. On a sample of 500 Spanish companies, the results indicate that the human resource practices carried out by family owned managed firms are generally less formalized and professionalized than those applied by externally family managed firms. Additionally, it has been found that the contribution of human resource practices through the selection process is negatively moderated by the family involvement
\end{abstract}

\footnotetext{
* Autor de contacto.
}

Correoselectrónicos: antonioc@um.es,gresanma@um.es, angelmer@um.es 


\section{Introducción}

La gestión de los recursos humanos en la empresa familiar es una tarea compleja, en un escenario en el que las relaciones entre propietarios, directivos, empleados y familia no están claramente delimitadas en términos de autoridad y responsabilidades (Leon-Guerrero et al., 1998; Reid et al., 2000). La influencia de la familia en las estructuras de gestión del capital humano crea un marco idiosincrásico, que hace diferente a la empresa familiar con respecto a la no familiar (Astrachan y Kolenko, 1994; Chrisman et al., 2005; Cruz et al., 2011), con posibles implicaciones tanto positivas como negativas en términos de desempeño organizativo, dependiendo de las preferencias que la empresa familiar muestre en relación con los intereses económicos del negocio o el bienestar de la familia (Schulze et al., 2001; Gomez-Mejia et al., 2007; Eddleston y Kellermans, 2007).

La revisión de la literatura desvela, en efecto, una diferenciación en el diseño de las prácticas de recursos humanos entre empresas familiares y no familiares según la preponderancia de objetivos económicos y/o no económicos. Por ejemplo, Reid y Adams (2001) y De Kok et al. (2006), examinando un conjunto de prácticas de recursos humanos selección, formación, desarrollo de carrera, evaluación y retribución-, encuentran que en las empresas familiares se aplican de forma menos formalizada que en la empresa no familiar, evitando criterios empresariales complejos. Asimismo, los hallazgos de los estudios que se han centrado en prácticas concretas de recursos humanos también son coherentes con estos planteamientos. Las empresas familiares suelen utilizar criterios de selección basados en el encaje de la cultura y valores de la organización (Gersick et al. 1997; Dyer y Montersen, 2006), poniendo más énfasis en acciones informales de formación para sus empleados y más orientadas a largo plazo (Kotey y Folker, 2007; Harris y Reid, 2008) y apostando por una retribución más tradicional y de carácter fijo, que apoye la equidad interna como criterio para recompensar y motivar a sus empleados (Anderson y Reeb, 2003; Carrasco-Hernandez y Sanchez-Marin, 2007).

Estos hallazgos apuntan, en principio, a que las prácticas de recursos humanos responden a la idiosincrasia de las empresas familiares. Con las decisiones adoptadas en recursos humanos se pretende optimizar una función de utilidad económica en las empresas no familiares, mientras que en las empresas familiares se busca un equilibrio entre el desempeño económico y no económico en el negocio (Gomez-Mejia et al, 2007). En el caso de las empresas familiares, este equilibrio viene motivado por la necesidad de implicar y comprometer social y emocionalmente al empleado para que contribuya a la supervivencia y preservación de la empresa familiar -o de los valores y cultura familiar en la empresa- (Berrone et al., 2010; Gomez-Mejia et al., 2011).

Sin embargo, varios aspectos ponen en duda estas evidencias. En primer lugar, no todos los estudios han evidenciado las relaciones en el mismo sentido. Así, por ejemplo, Miller et al. (2008) encuentran que las empresas familiares aplican, en mayor medida que las no familiares, ciertas prácticas "económicamente eficientes" de recursos humanos. Estos autores encuentran que las prácticas de formación adoptadas por las empresas familiares tienen mayor impacto económico y desarrollan más las habilidades específicas de los empleados. En segundo lugar, las dudas se acrecientan si se tiene en cuenta que, salvo contadas excepciones (Reid y Adams, 2001; De Kok et al., 2006), la mayoría de estudios han tratado las prácticas de recursos humanos en la empresa familiar de manera aislada, sin concebir un escenario conjunto en el que se consideren las interrelaciones entre prácticas por lo que los hallazgos, muchas veces, son circunstanciales y muy contextualizados. Por ejemplo, Cruz et al., (2010) señalan que las prácticas de selección de empleados en la empresa familiar, al ser habitualmente más adaptativas e informales, podrían producir efectos negativos ex-ante derivados de una selección adversa que minore la eficiencia de las subsiguientes prácticas de formación, desarrollo y retribución.

Poco se conoce, por tanto, acerca de si las prácticas de recursos humanos en la empresa familiar están más o menos orientadas a objetivos económicos frente a no económicos (continuidad y preservación de la riqueza familiar en la empresa) y cómo dicha orientación condiciona el mantenimiento de un capital humano eficiente que aporte al desempeño organizativo. En este sentido, ni siquiera las más recientes investigaciones meta-analíticas (Van Essen et al., 2011; Sacristan-Navarro et al., 2011) llegan a conclusiones definitivas sobre el efecto -positivo o negativo- sobre el desempeño de la orientación en la toma de decisiones de empresas familiares frente a no familiares, incluyendo los aspectos relacionados con la gestión de sus recursos humanos.

Por ello, este trabajo se enmarca en la necesidad de realizar nuevas investigaciones que contribuyan a 
incrementar el conocimiento en relación con las diferencias e interrelaciones de las principales prácticas de recursos humanos (selección, formación y retribución) en el contexto de la mayor o menor coincidencia de intereses de propiedad y dirección y los potenciales problemas asociados al predominio de la dimensión familiar y/o empresarial que se contemplan desde la teoría de la agencia (Schulze et al., 2001; Chrisman et al., 2004; Westhead y Howorth, 2006). La base empírica utilizada consta de una muestra de 500 empresas españolas, cuyo grado de implicación familiar ha sido analizado en dos niveles, comparando entre empresas familiares y no familiares en términos de propiedad $\mathrm{y}$ comparando entre empresas de dirección familiar y dirección externa en términos de dirección.

El trabajo se ha estructurado de forma habitual. En primer lugar se desarrollan los aspectos teóricos que nos llevan a la formulación de hipótesis. Posteriormente se describe la metodología empírica -muestra, medida de variables y análisis estadísticos- y se presentan los resultados para, por último, exponer y discutir las principales conclusiones del estudio.

\section{Marco teórico e hipótesis de investigación}

\subsection{Prácticas de recursos humanos en empresas familiares vs. no familiares}

Los escasos estudios empíricos que han analizado el conjunto de prácticas de recursos humanos, reconocen una distinta orientación entre empresas familiares y no familiares. Tan sólo Reid y Adams (2001) y De Kok et al. (2006) han abordado un análisis global, encuentran que en la empresa familiar las prácticas de selección, formación, desarrollo, evaluación y retribución se aplican de forma menos formalizada y profesionalizada, enfatizando principios de dirección espontáneos e improvisados, contrarios al uso de criterios específicos de selección, a la planificación de acciones formativas o al diseño de sistemas de retribución basados en el mérito.

Los estudios que comparan prácticas aisladas de recursos humanos entre empresas familiares y no familiares son mayoritarios y arrojan resultados similares. Así, con respecto a las prácticas de reclutamiento y selección, algunos estudios (Gersick et al., 1997; Aldrich y Langton, 1998) han puesto de manifiesto que las empresas familiares no se basan en criterios claros de reclutamiento y selección, utilizando básicamente a conocidos y familiares sobre la base de referencias personales. Las empresas familiares tienden, así, a basarse más fuertemente en redes sociales cercanas durante el proceso de reclutamiento, a la vez que aplican procesos de selección entre un pequeño conjunto de candidatos quiénes -habitualmente familiaressuelen participar de los valores y cultura de la empresa familiar (Dyer y Montersen, 2006), rechazando procesos masivos de reclutamiento y criterios de selección desde el punto de vista de la adaptación del empleado a las exigencias específicas del puesto de trabajo, algo más propio de las empresas no familiares.

Los investigadores también han examinado si las empresas familiares realizan más formación que las no familiares, así como el grado de planificación de las acciones formativas. Kotey y Foler (2007) encuentran que las empresas familiares -con independencia de su tamaño- ponen más énfasis en acciones informales de formación para sus empleados, y sólo en el caso de las empresas no familiares, las acciones formativas crecen en complejidad, formalización $\mathrm{y}$ orientación al desarrollo a medida que el tamaño empresarial aumenta. Las empresas familiares destacan, sin embargo, en las relaciones de desarrollo de empleados a través del mentoring espontáneo. En este sentido, Fiegener et al. (1996) y Harris y Reid (2008) confirman que los directores generales suelen transmitir la visión estratégica del negocio a través de relaciones muy estrechas e informales con el sucesor que son complementadas con un largo periodo de mentoring. En el caso de la empresa no familiar, estas relaciones están basadas en una planificación de la carrera profesional de los aspirantes apoyada en criterios de educación y experiencia en relación al puesto y en decisiones de promoción formales y objetivas.

La dimensión familia también ejerce una importante influencia en el diseño de los sistemas de recompensas. Por ejemplo, Carrasco-Hernandez y Sanchez-Marin (2007) muestran que el nivel retributivo de los empleados de empresas familiares es menor que el de empresas no familiares, mientras que la utilización de incentivos es mucho más frecuente en el caso de empresas no familiares. Las empresas familiares suelen poner más énfasis en la antigüedad como criterio para guiar las promociones y establecer los niveles retributivos, dando más peso a las recompensas no monetarias en lugar de utilizar componentes de retribución variable, especialmente cuando dichos incentivos suponen participación en la propiedad (Anderson y Reeb, 2003; Gomez-Mejia et al., 2001, 2003). Recompensar a los empleados en 
función de su antigüedad supone premiarles retributivamente a cambio de su lealtad a la empresa y a la familia y no necesariamente por hechos o consecuciones específicas que se vinculen con incentivos individuales y/o grupales asociados al rendimiento de los empleados (Davis y Harveston, 2001).

Hay algunos autores que no acaban de compartir estos principios. Así, Beehr et al. (1997) y Miller et al. (2008) encuentran que las empresas familiares aplican prácticas de formación y desarrollo más efectivas que las de las empresas no familiares, sobre todo en lo que respecta al desarrollo de habilidades de liderazgo y de visión de largo plazo de la empresa. Sin embargo, su perspectiva sí es coincidente con la de la búsqueda del compromiso de los empleados para con la continuidad del negocio familiar a través de prácticas menos formalizadas y estandarizadas, que responden a necesidades muy precisas e individualizadas.

En general, las evidencias empíricas ponen de manifiesto que las prácticas de recursos humanos en las empresas familiares son aparentemente menos profesionalizadas que las de las empresas no familiares. En este sentido, Gomez-Mejia et al. (2007) asocian esta orientación con la idea de la preservación de la riqueza familiar, desde la que se afirma que la principal preferencia de la empresa familiar en la atracción, retención, desarrollo y motivación de su capital humano se rige más por principios sociales y emocionales relacionados con la familia. Desde esta perspectiva, las empresas familiares no siempre persiguen maximizar su eficiencia económica, si eso supone poner en peligro objetivos como la reputación, la continuidad y la influencia familiar en el negocio, (Chua et al., 2009; Miller et al., 2008) lo que se traduce en la búsqueda, desarrollo y motivación de empleados comprometidos e implicados con los valores familiares de la empresa, que contribuyan a la preservación y mejora de las utilidades no económicas de la familia, a la vez que previenen posibles riesgos de supervivencia del negocio (Berrone et al., 2010; Cruz et al., 2011).

En términos de la teoría de la agencia (Jensen y Meckling, 1976; Fama y Jensen, 1983), esta menor formalización y profesionalización de las prácticas de recursos humanos en las empresas familiares frente a las no familiares podría explicarse desde la existencia de relaciones de parentesco, que hace disminuir las diferencias de interés principal-agente y fomenta el compromiso y el altruismo de las personas vinculadas a la empresa familiar (Schulze et al., 2001; Chua et al., 2009). Las conductas altruistas crean un sistema de auto-refuerzo de incentivos que alienta a los miembros de la empresa -propietarios, directivos y empleados- a ser más cuidadosos y desinteresados entre ellos, fomentando el sentimiento de propiedad colectiva, lo que favorece la implicación en el negocio, aumenta la comunicación y la cooperación, y enfatiza la orientación al largo plazo (Zahra, 2003; Eddleston y Kellermanns, 2007). La fuerte implicación en el negocio influyen en el diseño de las prácticas de recursos humanos en la línea de simplificarlo, ya que hace prescindibles el diseño de mecanismos de selección, formación, supervisión y motivación de los empleados (Gomez-Mejia et al., 2001; Chrisman et al., 2004).

Por lo tanto, se espera una relación negativa entre profesionalización de las prácticas de recursos humanos y el carácter familiar de la empresa, tanto desde la óptica de la presencia de la familia en la propiedad (familiares vs. no familiares) como en la dirección (familiares de dirección familiar vs. familiares de dirección externa).

H1. Existe una relación negativa entre el carácter familiar y la profesionalización de las prácticas de recursos humanos. Esta profesionalización: (a) es inferior en las empresas familiares respecto a las no familiares; (b) es inferior en las empresas familiares de dirección familiar respecto a las empresas familiares de dirección externa.

\subsection{Interrelaciones entre prácticas de recursos humanos y efecto en el desempeño organizativo.}

La lógica que subyace en la literatura de recursos humanos supone que las prácticas de recursos humanos pueden contribuir a la mejora del rendimiento económico de la empresa si el empleado posee los requisitos en conocimientos y habilidades y tiene la motivación necesaria para aplicarlos con la discrecionalidad precisa (Nishii et al., 2008; Jones et al., 2010). Recientes investigaciones han proporcionado evidencias empíricas que dan consistencia a los efectos sinérgicos y positivos que las interacciones entre prácticas de recursos humanos tienen sobre el desempeño organizativo cuando los empleados son capaces de obtener, desarrollar y aplicar con motivación los conocimientos y habilidades en coherencia con las particularidades de la empresa (Subramony et al., 2008; Crook et al., 2011).

La obtención y mejora de conocimientos y habilidades está primeramente relacionada con las 
prácticas de selección. En el contexto de la empresa familiar, desde la teoría de agencia (Daily y Dollinger, 1992; Schulze et al., 2001; Chrisman et al., 2004) se concibe que las decisiones de selección de empleados deben orientarse a alinear los intereses de los miembros de la empresa, incrementar su participación e implicación en el negocio, y evitar la aparición de conflictos que ocasionen posteriores ineficiencias organizativas. En este sentido, dos líneas paralelas de investigación presentan distintos argumentos en relación con la práctica de selección y sus interacciones con otras prácticas, así como sus posibles consecuencias para el desempeño organizativo desde la perspectiva de la posible alineación de intereses individuales y riesgos compartidos.

Un primer grupo de investigaciones sostiene que la utilización de redes sociales cercanas para el reclutamiento y la habitual existencia de relaciones de parentesco en los procesos de selección en la empresa familiar (tal y como se ha descrito en la hipótesis 1), disminuye las diferencias de interés principal-agente y fomenta el compromiso de las personas en la empresa familiar (Chrisman et al., 2004). La empresa familiar se dota de empleados con una fuerte implicación en el negocio y una orientación de largo plazo (Rosenblatt et al., 1985; Berrone et al., 2010), limitando el riesgo moral entre los miembros de la familia y reduciendo diferencias de objetivos y asimetrías de información (Lubatkin et al., 2007). Estos efectos positivos también influyen en otras prácticas de recursos humanos, ya que simplifican la gestión del negocio y convierten en innecesarios la implantación de formalizados mecanismos de supervisión y recompensa de los empleados (DeAngelo y DeAngelo, 1985; Leung, 2003).

La conducta altruista es el elemento clave en la explicación de los efectos positivos de la selección en el desempeño. El altruismo de los empleados, que se seleccionan por su cercanía con la cultura y valores de la empresa familiar, puede afectar positivamente a la relación entre prácticas de recursos humanos y desempeño organizativo al crear un sistema de auto-refuerzo de los incentivos que alienta a los miembros de la empresa a ser más cuidadosos y desinteresados en el desarrollo y aplicación esforzada de sus conocimientos y habilidades (Van den Berghe y Carchon, 2003). Asimismo, potencia el sentimiento de propiedad colectiva y de cooperación, lo que favorece la implicación en el negocio familiar y reduce las asimetrías de información (Eddleston y Kellermanns, 2007). Con la selección de este tipo de candidatos es más probable que aumente la alineación de intereses, contribuyendo a la preservación y mejora de las utilidades no económicas de la familia con relación al negocio (Berrone et al., 2010; Cruz et al., 2011). Contratar a alguien que es un experto en áreas de conocimiento especializado más allá de la comprensión de la familia propietaria podría aumentar el riesgo de aparición de asimetrías de información (GómezMejía et al., 2001), así como incrementar la incertidumbre de su comportamiento futuro, ya que la predicción para estos empleados se hace más difícil.

Un segundo grupo de estudios han identificado, sin embargo, un aumento de los conflictos de agencia en las empresas familiares, derivados precisamente de la inclusión de factores no económicos en la función de utilidad que la familia intenta maximizar, del altruismo mal entendido (conocido como altruismo asimétrico) entre los miembros de la familia y de la falta de auto-control entre los tomadores de decisiones (McConaughy, 2000; Schulze et al., 2001). Estos posibles problemas afectan directamente al diseño e implantación de las prácticas de recursos humanos.

Desde esta perspectiva, las empresas familiares sufren mayores problemas de selección adversa (que se produce cuando el candidato seleccionado no es el óptimo o más adecuado), originados, por un lado, por la masa de candidatos potenciales con menores conocimientos y habilidades que la empresa familiar suele atraer debido a las peores condiciones económico-laborales ofrecidas en comparación con la empresa no familiar (Schulze et al., 2003) y, por otro, por las concesiones en favor de la compatibilidad de los candidatos con la cultura y valores de la empresa familiar en detrimento de los conocimientos y habilidades del empleado (Jorissen et al., 2005; Chua et al., 2009).

Desde esta perspectiva se considera que el altruismo de la empresa familiar es asimétrico, constituyendo un factor que influye negativamente en la eficiencia de las prácticas de recursos humanos en términos de desempeño organizativo. Este altruismo mal entendido se genera como consecuencia del férreo control que realizan miembros de la familia sobre los asuntos empresariales (Schulze et al., 2003), de la posible incapacidad para atraer a los mejores empleados no familiares (Gómez-Mejía et al., 2001) y de la colocación de los miembros de la familia en puestos para los que no están suficientemente capacitados 
(Burkart et al., 2003). La consecuencia a nivel de prácticas de recursos humanos se traduce en

que si la empresa familiar no va ser capaz de seleccionar a los mejores, el efecto del resto de prácticas de recursos humanos sobre los empleados seleccionados no va a ser óptimo, generando menores utilidades a nivel de desempeño organizativo (Schulze et al., 2001, 2003).

En sentido contrario, en las empresas no familiares se ha evidenciado que a medida que la selección se fundamenta en criterios válidos y objetivos, aumenta la relación positiva entre ésta, la utilización de sistemas formalizados e intensivos de formación, la aplicación de prácticas retributivas basados en incentivos, generando todo ello un efecto positivo en el desempeño organizativo (Bowen y Ostroff, 2004; Azevedo y Akdere, 2011):.

Los argumentos anteriores nos permiten afirmar que, aunque existe cierta evidencia empírica que sugiere que el altruismo en la empresa familiar puede reducir los conflictos e incidir positivamente en el impacto de las prácticas de recursos humanos sobre el desempeño organizativo (Gomez-Mejia et al., 2001; Chrisman et al., 2004), también tiene graves consecuencias negativas (altruismo asimétrico) que pueden afectar a la eficiencia de las prácticas de recursos humanos (Schulze et al., 2001, 2003; Chua et al., 2009).

Si consideramos que, desde el punto de vista del desempeño económico, la implicación de la familia en el negocio hace que las prácticas de recursos humanos queden condicionadas por la selección adversa, cabe esperar que, por un lado, un efecto mediador de la misma en las prácticas de formación y retribución $y$, por otro, que a pesar de que las prácticas puedan contribuir a preservar la riqueza socioemocional de la familia (objetivo no económico) en el caso de la empresa familiar, tengan consecuencias negativas en el desempeño organizativo.

$\mathrm{H} 2$. La selección tiene un efecto mediador en la influencia de formación y retribución sobre el desempeño organizativo.

H3. Existe un efecto moderador negativo del carácter familiar en la influencia de las prácticas de recursos humanos sobre el desempeño organizativo. Esta influencia es: (a) inferior en las empresas familiares respecto a las no familiares; (b) inferior en las empresas familiares de dirección familiar respecto a las empresas familiares de dirección externa
Figura 1

Modelo de investigación

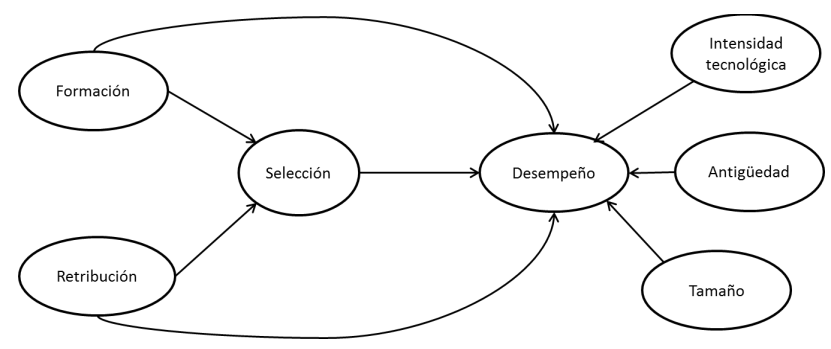

\section{Metodología}

\subsection{Muestra y recogida de datos}

Para contrastar empíricamente las hipótesis planteadas se han recogido datos de empresas españolas familiares y no familiares. La población inicial, seleccionada de la base OSIRIS (del Bureau Van Dyck Electronic Publishing), consiste en un total de 5113 empresas familiares y no familiares de todos los sectores industriales cuyo número de empleados se encuentra entre 25 y 249 . El diseño de la muestra es estratificado por tamaño y condición de empresa familiar. Se realizó una encuesta telefónica durante el primer trimestre de 2011, obteniendo información para 500 empresas (error muestral de $4,7 \%$ para $\mathrm{p}=\mathrm{q}=0,5$ y $95,5 \%$ de nivel de confianza), lo que supone un nivel de respuesta del 9,78\% porcentaje similar a otros estudios de este tipo. En la muestra final, 200 empresas entran en la categoría de pequeñas (entre 25 y 49 trabajadores) y 300 en la categoría de medianas (entre 50 y 249 trabajadores). Se han descartado a las empresas de menor tamaño, por su falta de formalización, así como por no tener un conjunto de prácticas de recursos humanos claramente predefinidas. Igualmente, se han descartado a empresas de gran tamaño por existir grandes diferencias de recursos respecto a las pequeñas y medianas empresas, lo que podría desvirtuar el análisis de las prácticas de recursos humanos. Finalmente, hay que señalar que la encuesta ha sido realizada al director de recursos humanos o, en su defecto, al director general de la empresa, debido a que su posición garantiza un conocimiento profundo de las políticas de personal cuyo estudio se aborda.

Aunque la selección de la muestra fue aleatoria, se analizó el sesgo de no respuesta. Siguiendo el razonamiento de Armstrong y Overton (1977), los últimos en responder son más similares a los que no responden. No se encontraron diferencias 
significativas en las variables del estudio entre los primeros y últimos en responder, sugiriendo así que no existe este sesgo.

La varianza del método común es una amenaza importante en la investigación conductual. Como sugiere Podsakoff et al. (2003) algunos investigadores recurren al análisis factorial confirmatorio. Un peor ajuste del modelo unidimensional sugeriría que la varianza del método común no es una amenaza. El modelo unidimensional alcanza una $\chi 2=1064.01$ con 54 grados de libertad (CFI $=0.633$; RMSEA $=0.194)$. Este ajuste es considerablemente peor que el modelo de medida, sugiriendo que no hay sesgo de método común en este estudio.

\subsection{Medidas y propiedades psicométricas de las escalas}

Prácticas de recursos humanos. Para la medición de la gestión de recursos humanos se ha creado una escala Likert formada por 9 ítemes -provenientes de la combinación de las escalas propuestas por Chen y Huang (2009) y de Thang y Quang (2005)- que miden las tres prácticas más importantes de la gestión de recursos humanos: selección (tres ítemes), formación (tres ítemes) y retribución (tres ítemes). Tal y como se han definido los ítemes, utilizando una escala likert de cinco puntos, se ha medido la orientación de cada una de las prácticas desde criterios poco formalizados y vinculados a las necesidades de la familia -valor 1- hasta criterios más formalizados y vinculada a objetivos empresariales -valor 5- (Carlson et al., 2006). En la tabla 1 aparece la formulación concreta de cada uno de los ítemes que componen la escala.

Desempeño organizativo. El uso de indicadores cuantitativos está extendido en la literatura debido a la creciente disponibilidad de información empresarial. Sin embargo, hay que señalar el creciente recurso a las variables cualitativas para medir los resultados organizativos puesto que son un sustituto razonable de las medidas objetivas (Dess y Robinson, 1984). Además puede señalarse que las empresas están más dispuestas a divulgar datos de tipo cualitativo; y porque facilita la comparación entre los resultados de distintas empresas, especialmente si pertenecen a diferentes sectores (Bae y Lawler, 2000). La medición del desempeño organizativo se ha basado en una escala de tres ítems subjetivos extraídos de Quinn y Rohrbaugh (1983) y Choi y Lee (2003) y formulados en una escala tipo Likert de cinco puntos, 1 (totalmente en desacuerdo) a 5 (totalmente de acuerdo), tal y como se muestra en la tabla 1.

Empresa familiar. El carácter familiar de la empresa se analiza en dos niveles: el primero, comparando las empresas familiares frente a las no familiares (propiedad) y, el segundo, considerando la implicación familiar en la dirección de la empresa (dirección). Con respecto al primero, el criterio de categorización de empresa familiar sobre la base de la propiedad se basa en la autoclasificación. Se ha preguntado al director general que califique a su empresa como familiar o no familiar en función de si la propiedad familiar es lo suficientemente significativa para controlar la empresa, tal y como apuntan diversos trabajos (Westhead y Cowling, 1998; Sonfield y Lussier, 2004). Se ha obtenido que el $54,4 \%$ de las empresas son familiares mientras que el $45,6 \%$ son no familiares. Con relación al segundo, el nivel de implicación familiar concierne exclusivamente a las empresas familiares y se mide a través de la participación de la familia en la dirección. La variable dirección familiar es clave en el campo de la empresa familiar, dado que es la dirección antes que la propiedad lo que determina la orientación hacia los resultados de la empresa (Astrachan et al., 2002; Westhead y Howorth, 2006; Sciascia y Mazzola, 2008). En la muestra de empresas familiares se ha realizado un análisis de conglomerados atendiendo a la participación de la familia en la dirección obteniendo dos grupos $(\mathrm{F}=1420,87 ; \mathrm{p}=0,000)$ : empresas familiares de dirección familiar $(\mathrm{n}=121)$ donde el promedio de participación de la familia en la dirección es el $92 \%$ y empresas familiares de dirección externa $(n=151)$, en este caso el promedio de participación de la familia en la dirección es el $20 \%$.

\section{Variables de control.}

Tamaño de la empresa. La mayoría de la literatura reconoce el tamaño de la empresa como una de las variables más importantes que condiciona las decisiones en la empresa familiar (Miller et al., 2000; Wasserman, 2006), incluidas aquellas relacionadas con la gestión de recursos humanos (Leon-Guerrero et al., 1998; Reid et al., 2000). Por ello, se ha incluido como variable de control, medida como el logaritmo neperiano del número de empleados promedio durante el año. Antigüedad, ha sido utilizada en varios estudios que relacionan las prácticas de recursos humanos y el desempeño (Delaney and Huselid, 1996), la medimos como número de años desde la fundación de la empresa. Intensidad tecnológica, variable que sirve para medir la influencia del sector de actividad. Algunos 
estudios han analizado su influencia tanto en las decisiones de recursos humanos como las de desempeño organizativo (Lepak et al., 2003). Siguiendo la clasificación de la OCDE se distinguen tres niveles desde sectores de baja intensidad a sectores de alta intensidad tecnológica.

Para comprobar las propiedades psicométricas de las escalas empleadas, llevamos a cabo un análisis factorial confirmatorio (CFA) utilizando el paquete fiabilidad estricta. Para evaluar la validez convergente aplicamos la varianza extraída media (AVE). También para este caso, todos los constructos presentan un AVE superior al valor 0,5 recomendado por Fornell y Larcker (1981). Los estadísticos del ajuste del modelo son satisfactorios $(\chi 2(48)=137,582 \mathrm{CFI}=0,967$ RMSEA $=0,061)$.

Para valorar la validez discriminante (Barclay et al., 1995), se recomienda el uso de la varianza

Tabla 1

Análisis factorial confirmatorio de las escalas de medida

\begin{tabular}{|c|c|c|c|}
\hline $\begin{array}{l}\text { Ítemes } \\
\text { Desempeño organizativo }\end{array}$ & Cargas factoriales & $\begin{array}{l}\text { Fiabilidad } \\
\text { compuesta } \\
0,977\end{array}$ & $\begin{array}{l}\text { AVE } \\
88,2 \%\end{array}$ \\
\hline Es más rentable & 0,767 & & \\
\hline Tiene un mejor comportamiento en ventas & 0,860 & & \\
\hline Presenta mayor independencia financiera & 0,709 & & \\
\hline Selección & & 0,977 & $88,2 \%$ \\
\hline La rigurosidad en el proceso de selección & 0,790 & & \\
\hline $\begin{array}{l}\text { La selección de empleados en función de habilidades } \\
\text { requeridas para el puesto }\end{array}$ & 0,786 & & \\
\hline $\begin{array}{l}\text { La selección de empleados según potencial de desarrollo en } \\
\text { la empresa }\end{array}$ & 0,756 & & \\
\hline Formación & & 0,993 & $92,7 \%$ \\
\hline La existencia de una política formativa integral & 0,824 & & \\
\hline La disponibilidad de formación continua para empleados & 0,928 & & \\
\hline $\begin{array}{l}\text { El fácil acceso de los nuevos contratados a acciones } \\
\text { formativas }\end{array}$ & 0,827 & & \\
\hline Retribución & & 0,940 & $82,7 \%$ \\
\hline $\begin{array}{l}\text { La estrecha relación entre el rendimiento del empleado y la } \\
\text { retribución que recibe }\end{array}$ & 0,729 & & \\
\hline El pago de incentivos individuales (bonus o primas) & 0,729 & & \\
\hline El pago de incentivos de grupo (reparto de beneficios) & 0,599 & & \\
\hline
\end{tabular}

SPSS Amos 19. Todas las cargas factoriales fueron significativas, lo que prueba la validez convergente de nuestros datos (Bagozzi y Yi, 1988). La fiabilidad compuesta fue calculada de acuerdo con Bagozzi y Yi (1988), mientras que la varianza extraída se hizo de acuerdo con las recomendaciones de Fornell y Larcker (1981). Como podemos observar en la tabla 2 , todos los constructos introducidos en el estudio superan el valor de 0,8 . Nunnally (1978) sugiere que valores superiores a 0.8 pueden considerarse de una extraída media (Fornell y Larcker, 1981). Para ello comparamos la raíz cuadrada del AVE (diagonal de la tabla 2) con las correlaciones entre los constructos (elementos fuera de la diagonal de la tabla 2). Como podemos observar, la raíz cuadrada del AVE es para todos los constructos mayor que la correlación entre ellos, lo que sugiere que cada constructo se relaciona más fuertemente con sus propias medidas que con otras. El análisis de correlaciones revela una elevada asociación entre el desempeño y la selección, siendo

Tabla 2

Descriptivos y análisis de validez discriminante de las escalas de medida.

\begin{tabular}{lrrllll}
\hline & Media & Desv.tip. & \multicolumn{1}{c}{$\begin{array}{c}\text { Desempeño } \\
\text { organizativo }\end{array}$} & Selección & Formación & Retribución \\
\hline $\begin{array}{l}\text { Desempeño } \\
\text { organizativo }\end{array}$ & 3,485 &, 878 & 0,939 & & & \\
Selección & 3,980 &, 853 & $0,224^{* * *}$ & 0,939 & & \\
Formación & 3,814 & 1,029 & $0,122^{* *}$ & $0,635^{* * *}$ & 0,963 & 0,940 \\
Retribución & 3,068 & 1,077 & $0,104^{*}$ & $0,299^{* * *}$ & $0,634^{* * *}$ & 0 \\
\hline
\end{tabular}

$$
* \mathrm{p}<0,1 ; * * \mathrm{p}<0,05 ; * * * \mathrm{p}<0,01
$$


Tabla 3.

ANOVA. Resultados para Resultados para Empresa Familiar y Empresa no Familiar; EF Dirección Familiar y EF Dirección Externa

\begin{tabular}{|c|c|c|c|c|c|c|}
\hline & & $\begin{array}{c}\text { Desempeño } \\
\text { organizativo }\end{array}$ & Tamaño & Selección & Formación & Retribución \\
\hline Empresa no Familiar & & 3.51 & 93.1 & 3.97 & 3.80 & 2.95 \\
\hline Empresa Familiar & & 3.47 & 72.1 & 3.99 & 3.83 & 3.16 \\
\hline $\mathrm{F}$ & & 0.251 & $12.180 * * *$ & 0.063 & 0.105 & $4.845^{* *}$ \\
\hline EF Dirección Externa & & 3.48 & 81.4 & 4.13 & 3.97 & 3.27 \\
\hline \multirow[t]{2}{*}{ EF Dirección Familiar } & & 3.42 & 60.5 & 3.80 & 3.64 & 2.99 \\
\hline & $\mathrm{F}$ & 0.289 & $6.403 * *$ & $10.1797 * * *$ & $6.694 * * *$ & $4.861 * *$ \\
\hline
\end{tabular}

algo menor con las otras dos prácticas, formación y retribución. Por otra parte, también se encuentran muy interrelacionadas las tres prácticas entre sí.

Con el objeto de profundizar en la relación entre las variables se realizó un análisis de la varianza cuyos resultados se muestran en la tabla 3 . No se producen diferencias en la variable desempeño organizativo ni por el carácter familiar ni dentro de las empresas familiares por el tipo de dirección. Las empresas no familiares tienen un mayor tamaño medido en número de empleados, lo mismo ocurre con las empresas familiares con dirección externa respecto a aquellas con dirección familiar. En cuanto a las prácticas de recursos humanos sólo se advierten diferencias significativas en una mayor puntuación en las empresas familiares en la práctica de retribución. Relativo a las diferencias entre empresas familiares, aquellas de dirección externa presentan valores significativamente superiores a las empresas de dirección familiar en las tres prácticas de recursos humanos. Por tanto, los datos evidencian que las mayores diferencias se producen entre empresas familiares atendiendo al nivel de implicación familiar en la dirección.

\section{Resultados}

Para el contraste de la primera hipótesis se recurre a un análisis multivariable de covarianzas (Mancova). Las variables dependientes son las prácticas de recursos humanos (selección, formación y retribución). El carácter familiar actúa como factor fijo, adicionalmente se ha incluido el tamaño como covariable para analizar su influencia sobre las variables dependientes. La principal ventaja de este análisis multivariable sobre el análisis de la varianza es que mide las influencias sobre las variables dependientes simultáneamente. Para la comparación entre empresas familiares frente a no familiares, en el análisis Mancova se obtiene un estadístico Lambda de Wilks de 0,987 apenas significativo $(p<0,1)$. En el caso de la comparación entre empresas familiares de dirección familiar frente a empresas familiares de dirección externa el estadístico es 0,965 siendo más significativo $(\mathrm{p}<0,05)$.

Las pruebas intersujetos se muestran en la tabla 4. Claramente se advierte que el modelo que analiza la influencia del carácter familiar atendiendo a si la

Tabla 4

MANCOVA inter-sujetos. Resultados para Empresa Familiar y Empresa no Familiar; EF Dirección Familiar y EF Dirección Externa

\begin{tabular}{lccc} 
& Selección & Formación & Retribución \\
Empresa Familiar vs. Empresa no Familiar & $2,753^{*}$ & $3,605^{* *}$ & \\
\hline Modelo & $4002,16^{* * *}$ & $2477,36 * * *$ & $4,648^{* * *}$ \\
Intersección & $5,419^{* *}$ & $7,040^{* * *}$ & $1456,19^{* * *}$ \\
Tamaño & 0,403 & 0,637 & $4,418^{* *}$ \\
Empresa Familiar vs. & & $6,220^{* *}$ \\
Empresa no Familiar & $5,909^{* * *}$ & $4,802^{* * *}$ & \\
EF Dirección Familiar vs. EF Dirección Externa & $2770,25^{* * *}$ & $1614,34 * * *$ & $3,964 * * *$ \\
Modelo & 1,598 & $2,864 *$ & $989,69 * * *$ \\
Intersección & $8,774 * * *$ & $5,300 * *$ & $3,209^{*}$ \\
Tamaño & & $3,490^{*}$ \\
EF Dirección Familiar vs. & & \\
EF Dirección Externa & & & \\
\hline
\end{tabular}


empresa es familiar presenta un peor ajuste que el que atiende a la implicación familiar. En el primero de los modelos (empresa familiar frente a empresa no familiar), los resultados muestran que el tamaño influye en la implantación de las prácticas de recursos humanos en general, y sólo en el caso concreto de la retribución influye el carácter familiar de la empresa. Las empresas familiares presentan unas prácticas de retribución más formalizadas. Así, no existe una relación negativa entre el carácter familiar de la empresa y la profesionalización de las prácticas de recursos humanos, tan sólo existe una relación con la implantación de prácticas de retribución y es positiva. Por tanto, se rechaza la hipótesis 1a relativa a que la profesionalización de las prácticas de recursos humanos es inferior en las empresas familiares que en las no familiares. El segundo modelo (empresa de dirección familiar vs. de dirección externa) pone de manifiesto la importancia de la dirección en las empresas familiares. La adopción de las prácticas de recursos humanos apenas se explica por el tamaño de la empresa sino por la presencia de directivos ajenos a la familia. Estos resultados confirman parcialmente la hipótesis 1, dado que sí se comprueba una relación negativa entre el carácter familiar y la profesionalización de las prácticas de recursos humanos, que ocurre fundamentalmente en las empresas familiares, según la implicación familiar en la dirección (H1b).

Las hipótesis 2 y 3 se contrastan a través de un modelo de ecuaciones estructurales, en el que la selección se sitúa como variable mediadora de las otras dos prácticas de recursos humanos (Figura 1). Existen diversas estrategias para estimar la significatividad de los efectos indirectos
(MacKinnon, Lockwood, Hoffman, West, \& Sheets, 2002; MacKinnon, Lockwood, \& Williams, 2004) entre las que se encuentra la técnica de bootsraping. Preacher, Rucker y Hayes (2007) describen esta técnica y justifican su uso para la estimación de efectos indirectos. Cheung y Lau (2007) demuestran que a través de ecuaciones estructurales se obtienen estimaciones insesgadas de mediación y los intervalos de confianza bootstraping corregidos de sesgo son los que mejor resultados proporcionan. Precisamente este será el planteamiento en esta investigación para analizar la significatividad de los efectos indirectos.

El modelo general que incluye todas las empresas (Tabla 5) muestra un ajuste correcto $(\mathrm{X} 2(84)=187.94 ; \quad \mathrm{CFI}=.962: \quad \mathrm{RMSEA}=.050)$. Las prácticas de recursos humanos influyen sobre el desempeño de la empresa. La selección directamente $(\beta=.200 ; p<.05)$. Las otras dos prácticas de forma indirecta a través de la selección, tanto la retribución $(\beta=.066 ; p<.05)$ como la formación $(\beta=.086 ; p<.05)$; no observándose influencia directa de ninguna de ellas. Por tanto se confirma la hipótesis de mediación de la selección.

Para estudiar el efecto del carácter familiar de la empresa se recurre al análisis multigrupo. Un primer análisis compara empresas no familiares con empresas familiares $(\mathrm{X} 2(168)=286.243$; $\mathrm{CFI}=.958$ : RMSEA =.038) y se analizan todas las relaciones incluyendo las relaciones directas entre formación y retribución sobre el desempeño. El segundo análisis divide a las empresas familiares en un grupo de dirección familiar $\mathrm{y}$ otro de dirección externa $(\mathrm{X} 2(168)=230.470 ; \quad \mathrm{CFI}=.960: \quad \mathrm{RMSEA}=.037)$, según el análisis cluster explicado en la definición

Tabla 5

Coeficientes modelos estructural

\begin{tabular}{|c|c|c|c|c|c|}
\hline \multirow{3}{*}{ Efectos directos } & \multirow{3}{*}{ Todas } & \multicolumn{2}{|c|}{ Empresa } & \multicolumn{2}{|c|}{ Dirección } \\
\hline & & Familiar & No Familiar & Familiar & Externa \\
\hline & & & & & \\
\hline Formación $\rightarrow$ Selección & $.427 * * *$ & $.415 * * *$ & $.464 * * *$ & $.662 * * *$ & $.265 * *$ \\
\hline Retribución $\rightarrow$ Selección & $.328 * * *$ & $.331 * * *$ & $.309 * * *$ & .090 & $.438 * * *$ \\
\hline Selección $\rightarrow$ Desempeño & $.200 * *$ & $.207 *$ & $.241 * *$ & .238 & $.231 *$ \\
\hline Formación $\rightarrow$ Desempeño & .007 & -.106 & .115 & -.076 & -.072 \\
\hline $\begin{array}{l}\text { Retribución } \rightarrow \text { Desempeño } \\
\text { Efectos indirectos }\end{array}$ & -.005 & .004 & -.016 & -.002 & -.069 \\
\hline Retribución $\rightarrow$ Desempeño & $.066^{* *}$ & $.069 * *$ & $.075 * *$ & .021 & $.101 *$ \\
\hline $\begin{array}{l}\text { Formación } \rightarrow \text { Desempeño } \\
\text { Variables de control }\end{array}$ & $.086^{* *}$ & $.086^{* *}$ & $.112 *$ & .158 & .061 \\
\hline Tamaño & .069 & $.141 * *$ & -.046 & $.166^{*}$ & $.157 *$ \\
\hline Antigüedad & .052 & -.018 & $.134 *$ & -.073 & .018 \\
\hline Intensidad tecnológica & -.034 & -.073 & .003 & -.002 & -.138 \\
\hline
\end{tabular}

$* \mathrm{p}<0,1 ; * * \mathrm{p}<0,05 ; * * * \mathrm{p}<0,01$ 
de la variable implicación familiar. Los resultados muestran que no se producen diferencias atendiendo a la propiedad, esto es, comparando resultados de las empresas familiares con las no familiares. En ambos casos se reproducen las relaciones del modelo general que incluye a todas las empresas. Sólo se distinguen por una mayor influencia no significativa de las prácticas de recursos humanos sobre el desempeño, tanto directamente (selección) como indirectamente (formación y retribución). También en la influencia de las variables de control, el tamaño contribuye al desempeño en las familiares mientras que en las no familiares es la antigüedad.

Atendiendo a la dirección, la comparación entre empresas familiares de dirección externa y aquellas de dirección familiar sí muestra diferencias más claras.

Las empresas familiares de dirección externa muestran un comportamiento más próximo a las empresas no familiares. La selección influye sobre el desempeño y también hay un efecto indirecto significativo de la retribución. En el caso de las empresas de dirección familiar no se produce influencia significativa de las prácticas de recursos humanos sobre el desempeño.

A la vista de los resultados, podemos confirmar la hipótesis 2 en la que se plantea el efecto mediador de la selección en la influencia de las prácticas de formación y retribución sobre el desempeño organizativo. Estas dos últimas prácticas en ningún caso tienen un efecto directo sobre el desempeño, dado que su influencia siempre se produce a través de la práctica de selección. La hipótesis 3, que plantea que el carácter familiar tiene un efecto moderador negativo en la influencia de las prácticas de recursos humanos sobre el desempeño, no se confirma totalmente, pues esto no ocurre en la comparación entre empresas familiares y no familiares.

En ambos casos, la influencia de las prácticas no se ve alterada por el carácter de propiedad familiar de la empresa. Sin embargo, sí existen diferencias dentro de las empresas familiares a nivel de dirección. Las empresas con una dirección externa a la familia siguen las pautas de las empresas familiares en la contribución de las prácticas de recursos humanos sobre el desempeño. No ocurre igual con las empresas de dirección familiar donde no existe una contribución significativa de las prácticas sobre el desempeño. Así, sólo se confirma la hipótesis $3 b$.

\section{Discusión y conclusiones}

Las prácticas de recursos humanos en la empresa familiar son idiosincrásicas, tomando distintas orientaciones en función de la complejidad de las relaciones entre propietarios, directivos y empleados de la organización en el contexto de la familia. Las preferencias de la familia propietaria en relación con los intereses económicos del negocio o el bienestar de la familia pueden determinar la eficiencia de las estructuras de gestión del capital humano (Miller et al., 2008; Cruz et al., 2011). Los pocos estudios empíricos que de forma más global han analizado estos aspectos así lo reconocen (Reid y Adams, 2001; De Kok et al., 2006), señalando claras diferencias de formalización y profesionalización de las prácticas de selección, formación, desarrollo, evaluación y retribución entre empresas familiares y no familiares. Son las empresas familiares las que optan por criterios más espontáneos e improvisados en el diseño y aplicación de sus prácticas de recursos humanos, mientras que las empresas no familiares se orientan por principios más formales y planificados en la gestión de sus empleados.

La investigación en esta línea, sin embargo, ha sido escasa, fragmentada y poco concluyente, con diferentes estudios proporcionando resultados poco consistentes como consecuencia de la diversidad de marcos teóricos, de definiciones de empresa familiar, de niveles de prácticas de recursos humanos y de consecuencias empresariales (Cruz et al., 2011; Gomez-Mejia et al., 2011). Por ello, con este trabajo se trata de contribuir a clarificar las diferencias entre la orientación de las prácticas de recursos humanos en empresas familiares, examinando las diferencias a nivel de propiedad empresas familiares vs. no familiares- y dirección empresas familiares de dirección familiar vs. empresas familiares de dirección externa-, a la vez que considerando los efectos interactivos entre prácticas de recursos humanos y su influencia en el desempeño organizativo. Utilizando el marco teórico que proporciona la teoría de la agencia (Chrisman et al., 2004; Chua et al., 2009), que proporciona un adecuado análisis de los factores económicos y sociales que determinan la orientación y eficiencia de las prácticas de recursos humanos, y sobre la base de una amplia muestra de 500 empresas industriales españolas, que ha permitido realizar comparaciones, dependiendo del nivel de propiedad y dirección familiar, entre 272 empresas familiares -145 de dirección familiar y 127 de dirección externa- y 228 no familiares, se han obtenido las siguientes conclusiones. 
En primer lugar, en contra de lo esperado, no se han encontrado diferencias entre la orientación de las prácticas de recursos humanos aplicadas por empresas familiares en comparación con empresas no familiares. En cambio, sí se ha confirmado que las prácticas de recursos humanos son significativamente diferentes entre empresas familiares según el tipo de dirección. Las empresas de dirección familiar adoptan unas prácticas de recursos humanas menos formalizadas y vinculadas a las necesidades familiares que las empresas familiares de dirección externa, que se caracterizan por un mayor nivel de profesionalización y de aplicación de principios empresariales en su gestión de recursos humanos. En la empresa de dirección familiar se opta por prácticas de selección basadas en criterios cercanos a la cultura y valores de la empresa familiar (Dyer y Montersen, 2006), se aplica una formación más informal y específica basadas en relaciones espontáneas (Kotey y Foler, 2007), y se retribuye en términos fijos recompensando la antigüedad más que el rendimiento como premio por la lealtad (Davis y Harverston, 2001).

Estos resultados son coherentes con algunas investigaciones recientes que señalan que no hay diferencias significativas en el comportamiento de la empresa familiar frente a no familiar si consideramos aspectos relacionados con la propiedad (Van Essen et al., 2011; SacristanNavarro et al., 2011). Dónde sí que aparecen los matices de gestión es en relación con la dimensión de dirección, tal y como señalan algunos autores en este sentido: la empresa es familiar cuando así lo consideran los directivos (Westhead y Cowling, 1998; Sonfield y Lussier, 2004). Es por ello que en las empresas familiares de dirección familiar, frente a las empresas familiares de dirección externa, se buscan prácticas que más que optimizar la eficiencia o el rendimiento económico, persiguen obtener, retener y desarrollar a aquellos trabajadores más comprometidos e implicados con los valores y la cultura de la empresa familiar. En términos de agencia, esta menor formalización y profesionalización de las prácticas de recursos humanos viene explicada por la elevada alineación de intereses principal-agente y el altruismo de las personas vinculadas a la empresa familiar (Schulze et al., 2001; Chua et al., 2009). En la empresa familiar de dirección familiar existe ya una fuerte implicación en el negocio que hace que no sean necesarios mecanismos adicionales de control y desarrollo de los recursos humanos (Gomez-Mejia et al., 2001; Chrisman et al., 2004), lo que explica la baja formalización y profesionalización de las prácticas.

En segundo lugar, nuestros resultados ponen de manifiesto, por un lado, la interrelación entre prácticas de recursos humanos y su efecto sinérgico (Subramony et al., 2008; Crook et al., 2011), al confirmar el efecto mediador que la selección, como primera práctica de recursos humanos, ejerce en la eficiencia de las prácticas de formación y retribución (Cruz et al., 2011) y, por otro, que este efecto es más o menos positivo en términos de desempeño organizativo en función del grado de implicación familiar en la empresa. En este caso, las diferencias se han producido tanto a nivel de propiedad como de dirección, por lo que la eficiencia de las prácticas de recursos humanos en términos de desempeño organizativo es menor tanto en las empresas familiares con relación a las no familiares, como en las empresas familiares de dirección familiar con respecto a las empresas familiares de dirección externa.

Estos resultados vuelven a confirmar los argumentos teóricos esgrimidos, desde los que se sostiene que las prácticas de selección son fundamentales para conseguir una adecuada alineación de intereses entre los miembros de la empresa familiar, aumentando de su identificación e implicación en el negocio, a la vez que se evitan conflictos y desajustes que ocasionen ineficiencias organizativas (Schulze, 2001; Chua et al., 2009). En las empresas familiares, la elevada alineación de intereses principal-agente limita el riesgo moral y reduce las asimetrías de información, haciendo innecesarios procesos de selección basados en criterios empresariales (Lubatkin et al., 2007). En su lugar, se utilizan criterios de selección más laxos e informales que se basen en la adaptación a la cultura y valores familiares que aseguren un elevado nivel de compromiso del empleado (DeAngelo y DeAngelo, 1985; Eddleston y Kellermanns, 2007), extendiéndose dichos criterios a la aplicación de las subsiguientes prácticas de formación y retribución.

Si bien esta orientación puede tener efectos positivos para la empresa familiar en términos no económicos -reputación, continuidad, etc.-, no compensan, al menos con la muestra que hemos manejado en este estudio, los efectos negativos que introduce el altruismo a través de la selección adversa y su influencia negativa en el resto de prácticas de formación y retribución (McConaughy, 2000; Jorissen et al., 2005). Este efecto negativo se produce de forma más significativa en las empresas familiares de dirección familiar, en las que la 
probabilidad de una mayor utilización de prácticas de selección más laxas y basadas en la adecuación a los valores familiares puede conducir a incurrir en costes ex-ante con efectos perjudiciales $-\mathrm{o}$ al menos nulos- en el desempeño organizativo, tanto directamente a través de la propia selección como indirectamente a través de las prácticas de formación y retribución (Burkart et al., 2003; Azevedo y Akdere, 2011).

En definitiva, en este trabajo se constata que la implicación de la familia en el negocio y la consiguiente introducción de objetivos no económicos, ejerce un claro efecto diferenciador en la gestión de recursos humanos de la empresa familiar con respecto a cualquier otro tipo de organización. Schulze et al. (2001) señala que las relaciones familia y empresa crean vínculos comunes y expectativas mutuas sustentadas en aspectos psicológicos distintos a los contractuales, en base a emociones, sentimientos y valores (confianza, altruismo, lealtad...) que impregnan el funcionamiento de la gestión de recursos humanos. En este sentido, nuestro estudio ha confirmado que la influencia de la familia en la orientación y eficiencia de las prácticas de recursos humanos se deriva de la dirección y no tanto de la propiedad dado que, como ya algunos estudios han señalado, es la dirección familiar antes que la propiedad es el factor que explica mejor la orientación hacia los resultados de la empresa (Westhead y Howorth, 2006; Sciascia y Mazzola, 2008). Además, se ha constatado que, en la disyuntiva de objetivos económicos y no económicos de la empresa familiar, son las prácticas de recursos humanos más profesionalizadas y con criterios empresariales las que mayor desempeño y por tanto, eficiencia económica, producen para la empresa. Esta orientación es la seguida mayoritariamente por las empresas familiares de dirección externa, sin embargo, no es el caso de las empresas familiares de dirección familiar, en las que podríamos suponer que hay una mayor presencia de objetivos relacionados con la riqueza y bienestar familiar más allá de lo meramente económico.

Finalmente, este trabajo no está exento de limitaciones, de las que somos conscientes y que pueden constituir, a su vez, líneas de investigación futuras. En primer lugar, sería deseable introducir en este debate la medición explícita de aspectos socioemocionales representativos de objetivos familiares, analizando su efecto en las prácticas de recursos humanos y en los resultados globales económicos y no económicos- de la empresa familiar, tal y como sugieren diversos estudios realizados sobre la base del reciente marco teórico de la preservación de la riqueza socioemocional de la empresa (Gomez-Mejia et al., 2007; Berrone et al., 2010; Cruz et al., 2011). En segundo lugar, las medidas de desempeño organizativo utilizadas únicamente se basan en escalas de percepción de la eficiencia organizativa por parte de los directivos. Aunque son útiles para determinados contextos y poseen ciertas ventajas (Bae y Lawler, 2000), sería deseable que fuesen completadas con indicadores objetivos de resultados extraídos de la información legal publicada por la empresa. Además, esta información podría ser más completa si se consiguiera complementar con otras medidas de resultados no económicos tales como reputación, representativas de los objetivos no económicos de la empresa familiar. Por último, se ha utilizado una medida clásica y sencilla de empresa familiar que, aunque aceptada y utilizada en la literatura, podría ser completada, no solo incluyendo propiedad y dirección, sino también aspectos relativos a la cultura y valores familiares -como la escala de FPEC- (Astrachan, et al. 2002)- que permitiera tener una perspectiva más global sobre las relaciones entre familia y negocio a nivel de gestión de recursos humanos.

\section{Bibliografía}

Aldrich, H., \& Langton, N. (1998). Human resource management practices and organizational life cycles. Frontier of entrepreneurship research. Babson Park, MA: Babson College Center for Entrepreneurial Studies.

Anderson, R.C., \& Reeb, D.M. (2003). Foundingfamily ownership and firm performance: Evidence from the S\&P 500. Journal of Finance, 58, 1301-1328.

Armstrong, J.S., Overton, T.S. (1977) Estimating nonresponse bias in mail surveys. Journal of Marketing Research 14, 396-402.

Astrachan, J., \& Kolenko, T. (1994). A neglected factor explaining family business success: human resource practices. Family Business Review, 7, 251-262.

Astrachan, J.; Klein, S.; Smyrnios, K. (2002) The FPEC Scale of Family Influence: A proposal for solving the business definition problem. Family Business Review, $15,45-58$.

Azevedo, R.E., \& Akdere, M. (2011). Examining agency theory in training \& development: Understanding self-interest behaviors in the organization. Human Resource Development Review, 10, 399-416.

Bae, J., \& Lawler, J.J. (2000). Organizational and HRM strategies in Korea: Impact on firm performance in an emerging economy. Academy of Management Journal, $43,502-517$. 
Bagozzi, R.P., \& Yi, Y. (1988). On the evaluation of structural equation models. Journal of the Academy of Marketing Science, 16, 74-94.

Barclay, D., Higgins, C., \& Thompson R. (1995). The partial least squares (PLS) approach to causal modelling: Personal computer adoption and use as an illustration. Technology Studies, 2, 285-309.

Beehr, T.A., Drexler, J.A. Jr., \& Faulkner, S. (1997). Working in small family businesses: empirical comparisons to non-family businesses. Journal of Organizational Behavior, 18, 297-312.

Berrone, P., Cruz, C., Gomez-Mejia, L.R., \& Larraza, M. (2010). Socio-emotional wealth and corporate responses to institutional pressures: Do family controlled firms pollute less? Administrative Science Quarterly, 55, 82-113.

Bowen, D.E., \& Ostroff, C. (2004). Understanding HRM-firm performance linkages: The role of "strength" of the HRM system. Academy of Management Review, 29, 203-221.

Burkart, M., Panunzi, F., \& Shleifer, A. (2003). Family-owned firms. Journal of Finance, 58, 2167-2201.

Carlson, D.S., Kacmar, M.K., Wayne, J.H., \& Grzywacz, J.G. (2006). Measuring the positive side of the work-family interface: development and validation of a work-family enrichment scale. Journal of Vocational Behavior, 68, 131-164.

Carrasco-Hernandez, A.J., \& Sanchez-Marin, G. (2007). The determinants of employee compensation in family firms: empirical evidence. Family Business Review, 20, 215-228.

Chandler, G., \& McEvoy, G. (2000). Human resource management, TQM, and firm performance in small and medium-sized enterprises. Entrepreneurship Theory and Practice, 25, 43-57.

Chen, C.J., \& Huang, J.W. (2009). Strategic human resource practices and innovation performance: The mediating role of knowledge management capacity. Journal of Business Research, 62, 104-114.

Cheung, G. \& Lau, R. (2008) Testing Mediation and Suppression Effects of Latent Variables Bootstrapping With Structural Equation Models. Organizational Research Methods, 11(2), 296-325.

Choi, B., \& Lee, H. (2003). An empirical investigation of KM styles and their effect on corporate performance. Information and Management, 40, 351-485.

Chrisman, J.J., Chua, J.H., \& Litz, R. (2004). Comparing the agency costs of family and nonfamilyowned firms: Conceptual issues and exploratory evidence. Entrepreneurship Theory and Practice, 28, 335-354.

Chrisman, J.J., Chua, J.H., \& Sharma, P. (2005). Trends and directions in the development of a strategic management theory of the family firm. Entrepreneurship Theory and Practice, 29, 555-575.
Chua, J.H., Chrisman, J.J., \& Bergiel, E.B. (2009). An agency theoretic analysis of the professionalized familyowned firm. Entrepreneurship Theory and Practice, 33, 355-372.

Crook, T.R., Todd, S.Y., Combs, J.G., Woehr, D.J., \& Ketchen, D.J. (2011). Does human capital matter? A meta-analysis of the relationship between human capital and firm performance. Journal of Applied Psychology, 96, 443-456.

Cruz, C., Firfiray, S., \& Gomez-Mejia, L.R. (2011). Socioemotional wealth and human resource management (HRM) in family-controlled firms. En Joshi, A., Liao, H., \& Martocchio, J.J. (Eds.): Research in Personnel and Human Resources Management, 30, 159-217. Emerald Group Publishing, Bingley.

Cruz, C., Gomez-Mejia, L.R., \& Becerra, M. (2010). Perceptions of benevolence and the design of agency contracts: CEO-TMT relationships in family firms. Academy of Management Journal, 53, 69-89.

Daily, C.M., \& Dollinger, M.J. (1992). An empirical examination of ownership structure in family and professionally managed firms. Family Business Review, 5, $117-136$.

Davis, P.S., \& Harveston, P.D. (2001). The phenomenon of substantive conflict in the family firm: A cross-generational study. Journal of Small Business Management, 39, 14-30.

De Kok, J., Uhlaner, L., \& Thurik, A. (2006). Professional HRM practices in family owned-managed enterprises. Journal of Small Business Management, 44, 441-460.

DeAngelo, H., \& DeAngelo, L. (1985). Managerial ownership of voting rights: A study of public corporations with dual classes of common stock. Journal of Financial Economics, 14, 33-69.

Delaney, J. T., \& Huselid, M.A. 1996. The impact of human resource management practices on performance in for-profit and nonprofit organizations. Academy of Management Journal, 39, 949-969.

Dess, G.G., \& Robinson, R.B. (1984). Measuring organizational performance in the absence of objective measures: the case of the privately-held firm and conglomerate business unit. Strategic Management Journal, 5, 265-273.

Dyer, W.G., Jr., \& Mortensen, S.P. (2006). Entrepreneurship and family business in a hostile environment: The case of Lithuania. Family Business Review, 18, 247-258.

Eddleston, K.A., \& Kellermanns, F.W. (2007). Destructive and productive family relationships: A stewardship theory perspective. Journal of Business Venturing, 22, 545-565.

Fama, E., \& Jensen, M. (1983). Separation of ownership and control. Journal of Law and Economics, 


\section{6, 301-325.}

Fiegener, M.K., Brown, B.M., Prince, R.A., \& File, K.M. (1996). Passing on strategic vision. Journal of Small Business Management, 34, 15-26.

Fornell, C., \& Larcker, D.F. (1981). Evaluating structural equation models with unobservable variables and measurement error. Journal of Marketing Research, $18,39-50$.

Gersick, K.E., Davis, J.A., Hampton, M., \& Lansberg, I. (1997). Generation to generation: life cycles of the family business. Harvard Business School Press, Boston.

Gomez-Mejia, L.R., Cruz, C., Berrone, P., \& De Castro, J. (2011). The bind that ties: Socioemotional wealth preservation in family firms. Academy of Management Annals, 5, 653-707.

Gomez-Mejia, L.R., Haynes, K., Nuñez-Nickel, M., Jacobson, K.J.L., \& Moyano-Fuentes, J. (2007). Socioemotional wealth and business risks in familycontrolled firms: Evidence from Spanish olive oil mills. Administrative Science Quarterly, 52, 106-137.

Gomez-Mejia, L.R., Larraza-Kintana, M., \& Makri, M. (2003). The determinants of executive compensation in family-controlled public corporations. Academy of Management Journal, 46, 226-237.

Gomez-Mejia, L.R., Nuñez-Nickel, M., \& Gutierrez, I. (2001). The role of family ties in agency contracts. Academy of Management Journal, 44, 81-95.

Habbershon, T.G, Williams. M., \& Macmillan, I.C. (2003). A unified systems perspective of family firm performance, Journal of Business Venturing, 18, 451-465.

Hall. A., \& Nordqvist, M. (2008). Professional management in family business: toward an extended understanding. Family Business Review, 21, 51-69.

Harris, R., \& Reid, R. (2008): Barriers to growth in family-owned smaller businesses. En Barret, R., \& Mayson, S. (Eds.): International handbook of HRM entrepreneurship, pp. 260-284. Dewuard Elgar, Cheltenham.

Jensen, M.C., \& Meckling, W.H. (1976). Theory of the firm: Managerial behavior, agency costs and ownership structure. Journal of Financial Economics, 3, 305-360.

Jones, D.C., Kalmi, P., \& Kauhanen, A. (2010). How does employee involvement stack up? The effects of human resource management policies on performance in a retail firm. Industrial Relations, 49 (1), 1-21.

Jorissen, A., Laveren, E., Martens, R., \& Reheul, A. (2005). Real versus sample-based differences in comparative family business research. Family Business Review, 18, 229-246.

Kanuk, L., Berenson, C. (1975) Mail surveys and response rates: a literature review. Journal of Marketing Research 22, 440-453.
Kotey, B., \& Folker, C. (2007). Employee training in SMEs: effect of size and firm type-family and nonfamily. Journal of Small Business Management, 45, 214-238.

Kuvaas, B. (2007). An exploration of how the employee-organization relationship affects the linkage between perception of developmental human resource practices and employee outcomes. Journal of Management Studies, 45, 1-25.

Leon-Guerrero, A., McCann III, E., \& Haley, J. (1998). A study of practice utilization in family businesses. Family Business Review, 11, 107-120.

Lepak, D.P., Takeuchi, R., \& Snell, S.A. (2003). Employment Flexibility and Firm Performance: Examining the Interaction Effects of Employment Mode, Environmental Dynamism, and Technological Intensity, Journal of Management, 29, 681-703.

Leung, A. (2003). Different ties for different needs: Recruitment practices of entrepreneurial firms at different developmental phases. Human Resource Management, $42,303-320$.

Lubatkin, M.H., Durand, R., \& Ling, Y. (2007). The missing lens in family-owned firm governance theory: A self-other typology of parental altruism. Journal of Business Research, 60, 1022-1029.

Lubatkin, M.H., Schulze, W.S., Ling, Y., \& Dino, R. N. (2005). The effects of parental altruism on the governance of family-managed firms. Journal of Organizational Behavior, 26, 313-330.

MacKinnon, D. P., Lockwood, C. M., \& Williams, J. (2004). Confidence limits for the indirect effect: Distribution of the product and resampling methods. Multivariate Behavioral Research, 39, 99-128.

MacKinnon,D. P., Lockwood, C. M., Hoffman, J. M.,West, S. G., \& Sheets, V. (2002).A comparison of methods to test mediation and other intervening variable effects. Psychological Methods, 7, 83-104.

McConaughy, D.L. (2000). Family CEOs vs. Nonfamily CEOs in the family-controlled firm: an examination of the level and sensitivity of pay to performance. Family Business Review, 13, 121-131.

Miller, D., Le Breton-Miller, I., \& Scholnick, B. (2008). Stewardship vs. stagnation: An empirical comparison of small family and non-family businesses. Journal of Management Studies, 45: 51-78.

Miller, N.J., Winter, M., Fitzgerald, M. A., \& Paul, J. (2000). Family microenterprises: Strategies for coping with overlapping family and business demands. Journal of Developmental Entrepreneurship, 5(2), 87-113.

Nishii, L.H., \& Lepak, D.P., \& Schneider, B. (2008). Employee attributions of the "why" of HR practices: Their effects on employee attitudes and behaviors, and customer satisfaction. Personnel Psychology, 61, 503545 .

Nunnally, J.C. (1978). Psychometric theory. New 
York: McGraw-Hill.

Oppenheim, A.N. (1966) Questionnaire Design and Attitude Measurement. Basic Books, New York.

Pearson, A., Carr, J., \& Shaw, J. (2008). Toward a theory of familiness: A social capital perspective. Entrepreneurship Theory and Practice, 32, 949-969.

Podsakoff, P.M; MacKenzie, S.B.; Lee, J-Y y Podsakoff, N.P. (2003). Common method biases in behavioural research: a critical review of the literature and recommended remedies, Journal of Applied Psychology, 88(5), 879-903.

Preacher, K.; Rucker, D. \& Hayes, A. (2007) Addressing Moderated Mediation Hypotheses: Theory, Methods, and Prescriptions, Multivariate Behavioral Research, 42:1, 185-227

Quinn, R.E., \& Rohrbaugh, J. (1983). A spatial model of effectiveness criteria: towards a competing values approach to organizational analysis. Management Science, 29, 363-377.

Reid, R., \& Adams, J. (2001). Human Resource Management: A survey of practices within family and non-family firms. Journal of European Industrial Training, 6, 310-320.

Reid, R., Morrow, T., Kelly, B., Adams, J., \& McCartan, P. (2000). Human resource management practices in SMEs: A comparative analysis of family and non-family-owned firms. Irish Business and Administration Research, 21, 157-181.

Rosenblatt, P.C., De Mik, L., Anderson, R.M., \& Johnson, P.A. (1985). The family in business. JosseyBass, San Francisco.

Sacristan-Navarro, M., Gomez-Anson, S., \& CabezaGarcia, L. (2011). Family ownership and control, the presence of other large shareholders, and firm performance: further evidence. Family Business Review, 24, 71-93.

Schulze, W.S., Lubatkin, M.H., \& Dino, R.N. (2003). Exploring the agency consequences of ownership dispersion among the directors of private family-owned firms. Academy of Management Journal, 46, 174-194.

Schulze, W.S., Lubatkin, M.H., Dino, R.N., \& Buchholtz, A.K. (2001). Agency relationships in family firms: theory and evidence. Organizational Science, vol. 12, 99-116.

Sciascia, S., \& Mazzola, P. (2008). Family involvement in ownership and management: Exploring nonlinear effects on performance. Family Business
Review, 21, 331 - 345.

Sharma, P., Chrisman, J., \& Chua, J. (1997). Strategic management of the family business: Past research and future challenges. Family Business Review, 10, 1-35.

Sonfield, M.C., \& Lussier, R.N. (2004). First-, second-, and third-generation family firms: A comparison. Family Business Review, 17, 189-202.

Steward, K. A., Segars, A. H. (2002). An empirical examination of the concern for information privacy instrument. Inform. Systems Res. 13(1), 36-49.

Straub, D. (1989). Validating instruments in MIS research. MIS Quarterly, 13(2) 147-169.

Subramony, M., Krause, N., Norton, J., \& Burns, G.N. (2008). The relationship between human resource investments and organizacional performance: A firmlevel examination of equilibrium theory. Journal of Applied Psychology, 93, 778-788.

Thang, L.C., \& Quang, T. (2005): Antecedents and consequences of dimensions of human resource management practices in Vietnam. International Journal of Human Resource Management, 16, 1830-1846.

Van den Berghe, L.A.A., \& Carchon, S. (2003). Agency relations within the family business system: An exploratory approach. Corporate Governance: An International Review, 11, 171-179.

Van Essen, M., Carney, M., Gedajlovic, E., Heugens, P., \& Van Oosterhout, J.H. (2011). How do family firms differ? A meta-analysis. Academy of Management Journal, 54, 437-460.

Wasserman, N. (2006). Stewards, agents, and the founder discount: executive compensation in new ventures. Academy of Management Journal, 49, 960-976.

Werner S., Tosi H.L., \& Gomez-Mejia L. (2005). Organizational governance and employee pay: how ownership structure affects the firm's compensation strategy. Strategic Management Journal, 26: 377-384.

Westhead, P., \& Cowling, M. (1998). Family firm research: The need for a methodological rethink. Entrepreneurship Theory and Practice, 23, 31-56.

Westhead, P., \& Howorth, C. (2006). Ownership and management issues associated with familiy firm performance and company objectives. Familiy Business Review, 19 (4), 301-316.

Zahra, S.A. (2003). International expansion of U.S. manufacturing family businesses: The effect of ownership and involvement. Journal of Business Venturing, 19, 495512. 